\title{
A FUZZY LOGIC INFERENCE APPROACH FOR THE ESTIMATION OF THE PASSENGERS FLOW DEMAND
}

\author{
Aránzazu Berbey Alvarez, Rony Caballero George \\ Universidad Tecnológica de Panamá \\ aranzazu.berbey@utp.ac.pa, rony.caballero@utp.ac.pa \\ Juan de Dios Sanz Bobi, Ramón Galán López \\ Universidad Politécnica de Madrid \\ juandedios.sanz@upm.es,ramon.galan@upm.es,
}

Keywords: $\quad$ Fuzzy logic inference, passenger flow modelling, maximum entropy theory, matrix method of area trips OD.

\begin{abstract}
This paper presents a new approach that designs the flow of passengers in mass transportation systems in presence of uncertainties. One of the techniques used for the prediction of passenger demand is the origindestination matrices. However, this method is limited to urban areas and rarely to explicit stations. Otherwise, the gravity models based on friction functions can be another alternative; however, it is difficult to fit into practical achievements. Another solution might be the application of artificial intelligence techniques so as to include some intuitive knowledge provided by an expert to predict the flow demand of passengers' trips in explicit stations. This paper proposes to combine a matrix of origin-destination trips of travel zones, with the intuitive knowledge, applying a fuzzy logic inference approach.
\end{abstract}

\section{INTRODUCTION}

The passenger flow modelling has been tackled by several authors (Kiluchi et al, 1999) (Aldian et al, 2003) (Watson and Prevedouros, 2006) (Cheng et al, 2009) (Murat, 2010) (Xie et al, 2010). Some of them have applied linear programming methods considering small samples (Murat, 2010). It has also been applied the maximum entropy theory (Xie et al, 2010) and results have been compared between different methods (Watson and Prevedouros, 2006). However, it has been shown that these methods have limitations in certain scenarios. In this case, the use of fuzzy logic has proven to be a promising tool because it can integrate the railway planning experts' experience in multiple scenarios (Aldian et al, 2003) (Cheng et al, 2009). This paper proposes to integrate the effectiveness of the methods based on origin-destination matrices, with the experience of experts in railway planning using an artificial intelligence support based on fuzzy logic.

\section{AREA ANALYSIS}

The matrix method of area trips O-D is one of the most used methods to design the movement of passengers. This method divides the urban environment in areas of interests that generate and attract trips. However, due to economic and practical reasons, the size of the interests' zones is usually large in order to apply directly to urban planning models. Therefore, it is interesting in many cases to divide these macro areas into smaller areas, allowing a better analysis of passenger flow.

\section{SUBZONE DESIGN}

Considering the attraction vectors $D_{T} \in \mathbb{R}^{1 \times n_{m}}$ and the generation vectors $O_{T} \in \mathbb{R}^{n_{m} \times 1}$, each element of the attraction vectors $D_{T J}$ and generation $O_{T I}$ can be subdivided into $n_{J}$ subzones, becoming two new 
vectors $D \in \mathbb{R}^{1 \times n_{s}}$ and $O \in \mathbb{R}^{n_{s} \times 1}$, where $n_{s}=\sum_{J=1}^{n_{m}} n_{J}$.

Therefore, the macro areas matrix $T \in \mathbb{R}^{n_{m} \times n_{m}}$ is subdivided at the same time, obtaining sub-zone matrix $M \in \mathbb{R}^{n_{s} \times n_{s}}$. Consequently, it can be shown that every element of the attraction and the generation vectors, as well as the macro area and the subzone matrices must meet with:

$$
\begin{gathered}
D_{T J}=\sum_{j=1+m_{B}(J)}^{m_{E}(J)} D_{j} \\
O_{T I}=\sum_{i=1+m_{B}(I)}^{m_{E}(I)} O_{i} \\
T_{I J}=\sum_{i=1+m_{B}(I)}^{m_{E}(I)} \sum_{j=1+m_{B}(J)}^{m_{E}(J)} M_{i j} \\
A_{j}=\sum_{i=1}^{n_{s}} M_{i j} \forall j=1 \cdots n_{s} \\
B_{i}=\sum_{j=1}^{n_{s}} M_{i j} \forall i=1 \cdots n_{s}
\end{gathered}
$$

where

$$
\begin{gathered}
m_{B}(x)=\sum_{K=1}^{x} n_{K-1} \\
m_{E}(x)=\sum_{K=1}^{x} n_{K} \\
n_{0}=0
\end{gathered}
$$

To obtain a subzone model, a system is governed by the principle of maximum entropy would be applied. However, this model can be improved by incorporating information by an expert, even if this is inaccurate; this supposition is done with the aim to improve the approximation, and it means to apply the principle of maximum entropy. The information can be included using techniques based on artificial intelligence and more specifically based on fuzzy logic.

We have to apply the following steps to set up the subzone model:

a) $\boldsymbol{T}_{I J}, \boldsymbol{O}_{T I}$ and $\boldsymbol{D}_{T J}$ have little uncertainty within the same planning horizon. b) Every element of the vector $\boldsymbol{O}_{\boldsymbol{i}}$ and $\boldsymbol{D}_{\boldsymbol{j}}$, corresponding to a subzone $\boldsymbol{i}$ or $\boldsymbol{j}$, that is part of the macro area $\boldsymbol{I}$ or $\boldsymbol{J}$ respectively, can be represented as a function of $\mathrm{O}_{T I}$ or $\boldsymbol{D}_{T J}$ vectors of the macro area in which it is contained, and by a potential function with exponents $\boldsymbol{C}_{\boldsymbol{D} \boldsymbol{J}}$ or $\boldsymbol{C}_{\boldsymbol{O} \boldsymbol{i}}$.

$$
\begin{gathered}
D_{j}=\frac{2^{\frac{1}{\ln 2} C_{D j}}}{n_{J} \sum_{k=1+m_{B}(J)}^{m_{E}(J)} 2^{\frac{1}{\ln 2} C_{D k}}} D_{T J} \\
O_{i}=\frac{2^{\frac{1}{\ln 2} C_{O i}}}{n_{I} \sum_{k=1+m_{B}(J)}^{m_{E}(J)} 2^{\frac{1}{\ln 2} C_{O k}}} O_{T I}
\end{gathered}
$$

c) Every element of the trip matrix $\boldsymbol{M}_{i j}$ corresponding to the subzones $\boldsymbol{i}, \boldsymbol{j}$ and that is part of the macro area $\boldsymbol{I}, \boldsymbol{J}$ respectively, can be represented as a function of $\boldsymbol{T}_{\boldsymbol{I J}}$ and by a potential function with exponent $\boldsymbol{C}_{\boldsymbol{M i j}}$.

$$
M_{i j}=\frac{2^{\frac{1}{\ln 2} C_{M i j}}}{n_{I} n_{J} \sum_{r=1+m_{B}(I)}^{m_{E}(I)} \sum_{s=1+m_{B}(J)}^{m_{E}(J)} 2^{\frac{1}{\ln 2} C_{M r s}}} T_{I J}
$$

d) $\boldsymbol{C}_{\boldsymbol{D} \boldsymbol{j}}, \boldsymbol{C}_{\boldsymbol{O} \boldsymbol{i}}$ and $\boldsymbol{C}_{M i j}$ come from a fuzzy inference engine based on the experience of an expert.

It is important to note that the exponents $\boldsymbol{C}_{\boldsymbol{D j}}$ and $\boldsymbol{C}_{\boldsymbol{O} \boldsymbol{i}}$ are related to the relative level of importance of the station in a given planning horizon. If these exponents are zero, the estimation becomes the estimation by maximum entropy, whereas if you have a negative or positive number it corresponds to a station with low or high demand respectively.

On the other hand the exponent $\boldsymbol{C}_{\boldsymbol{M} i j}$ establishes the relative level of importance of the passenger flow between two stations. Here, the estimation using the maximum entropy also corresponds for a value equal to zero, and a negative or positive number corresponds to a flow of low or high demand respectively. 


\section{FUZZY INFERENCE ENGINE}

The exponents $\boldsymbol{C}_{\boldsymbol{D} \boldsymbol{j}}, \boldsymbol{C}_{\boldsymbol{O} \boldsymbol{i}}$ and $\boldsymbol{C}_{\boldsymbol{M} \boldsymbol{i j}}$ can be designed using the help of fuzzy logic. In this particular case it seems reasonable to use triangular membership functions (Figure 1). These exponents can be estimated not only considering the proximity of the station by major population centres, such as: residential areas, industrial estates, hospitals, schools and shopping centres, but also with the presence of transport interchanges.

\begin{tabular}{|c|c|}
\hline VL & Very Low \\
\hline L & Low \\
\hline M & Medium \\
\hline H & High \\
\hline VH & Very High \\
\hline
\end{tabular}

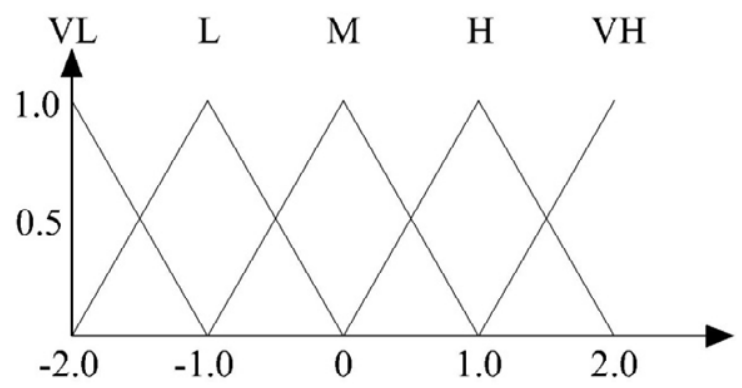

Figure 1: Starting fully logic functions for $\boldsymbol{C}_{D j}, \boldsymbol{C}_{\boldsymbol{O} i}$ y $\boldsymbol{C}_{\boldsymbol{M i j}}$.

\section{PROPOSED ARGORITHM}

a) Estimate the exponents $\boldsymbol{C}_{\boldsymbol{D j}}, \boldsymbol{C}_{\boldsymbol{O} \boldsymbol{i}}$ and $\boldsymbol{C}_{\boldsymbol{M} \boldsymbol{i j}}$ basing on the inaccurate available information.

b) Estimate the trip attraction and generation vectors $\boldsymbol{D}$ and $\boldsymbol{O}$, for the stations using,

$$
\begin{gathered}
D_{j}=\frac{2^{\frac{1}{\ln 2} C_{D j}}}{n_{J} \sum_{k=1+m_{B}(J)}^{m_{E}(J)} 2^{\frac{1}{\ln 2} C_{D k}}} D_{T J} \\
O_{i}=\frac{2^{\frac{1}{\ln 2} C_{O i}}}{n_{I} \sum_{k=1+m_{B}(J)}^{m_{E}(J)} 2^{\frac{1}{\ln 2} C_{O k}}} O_{T I}
\end{gathered}
$$

c) Estimate the trip matrix $\boldsymbol{M}_{\boldsymbol{i j}}$, using,

$$
M_{i j}=\frac{2^{\frac{1}{\ln 2} C_{M i j}}}{n_{I} n_{J} \sum_{r=1+m_{B}(I)}^{m_{E}(I)} \sum_{s=1+m_{B}(J)}^{m_{E}(J)} 2^{\frac{1}{\ln 2} C_{M r s}}} T_{I J}
$$

d) Thus it is checked if $O_{i}=\sum_{j=1}^{n_{s}} M_{i j} \forall i=1 \cdots n_{s}$. Consequently, if $\left|\sum_{j=1}^{n_{s}} M_{i j}-O_{i}\right|<\varepsilon$ is met for a given value, it is accepted. Meanwhile, if not, the values of the matrix elements are corrected with

$$
M_{i j} \Leftarrow \frac{O_{i} M_{i j}}{\sum_{j=1}^{n_{s}} M_{i j}}
$$

e) It is checked if $D_{j}=\sum_{i=1}^{n_{s}} M_{i j} \forall j=1 \cdots n_{s}$. Consequently, if $\left|\sum_{i=1}^{n_{s}} M_{i j}-D_{j}\right|<\varepsilon$ is met, it is accepted. Meanwhile, if not, the values of the matrix elements are corrected with

$$
M_{i j} \Leftarrow \frac{D_{j} M_{i j}}{\sum_{i=1}^{n_{s}} M_{i j}}
$$

f) It is checked if $T_{I J}=\sum_{i=1+m_{B}(I)}^{m_{E}(I)} \sum_{j=1+m_{B}(J)}^{m_{E}(J)} M_{i j}$ for all $i, \quad j, \quad I, \quad J . \quad$ Consequently if, $\left|\sum_{i=1+m_{B}(I)}^{m_{E}(I)} \sum_{j=1+m_{B}(J)}^{m_{E}(J)} M_{i j m}-T_{I J}\right|<\varepsilon_{m}$ is met, it is accepted. Meanwhile, if not, the values of the matrix elements are corrected with

$$
M_{i j} \Leftarrow \frac{T_{I J} M_{i j}}{\sum_{i=1+m_{B}(I)}^{m_{E}(I)} \sum_{j=1+m_{B}(J)}^{m_{E}(J)} M_{i j}}
$$




\section{RESULTS FOR SIMULATED DATA}

The following case illustrates how the proposed algorithm works. Let's consider the railway network topology shown in Figure 2. In this case it is known from previous studies that the mobility preferences among three regions so-called $\mathrm{A}, \mathrm{B}$ and $\mathrm{C}$, but without giving any specific details concerning the connection preferences between specific stations. The region $\mathrm{A}$ has the stations E1, E2 and E3; the region $\mathrm{B}$ has the stations $\mathrm{E} 4, \mathrm{E} 5, \mathrm{E} 6$ and $\mathrm{E} 7$; and the region $\mathrm{C}$ has only two stations $\mathrm{E} 8$ and $\mathrm{E} 9$.

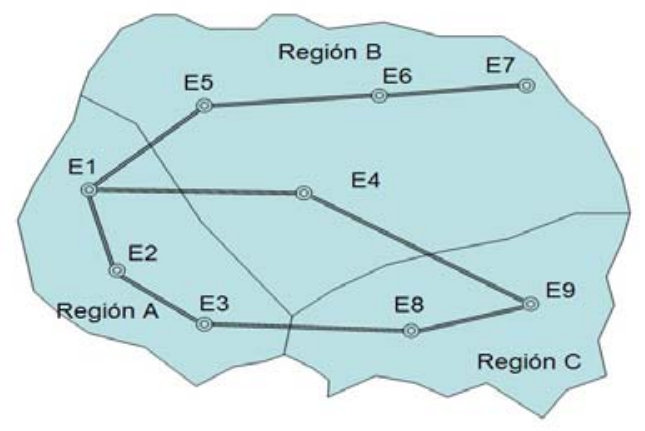

Figure 2: Red topology.

$\mathrm{O}-\mathrm{D}$ matrix between these regions is known and it is described below:

Table 1: O-D matrix between the regions.

\begin{tabular}{|c|c|c|c|c|}
\hline & A & B & C & \\
\hline A & 9397 & 5282 & 5213 & 19892 \\
\hline B & 25118 & 8272 & 5065 & 38455 \\
\hline C & 22570 & 8732 & 1134 & 32436 \\
\hline & 57085 & 22286 & 11412 & 90783 \\
\hline
\end{tabular}

We have to consider the estimated subzones exponents' vectors

$$
\begin{aligned}
& C_{D}=\left[\begin{array}{lllllllll}
A & A & M & A & M & M & M & M A & B
\end{array}\right], \\
& C_{o}=\left[\begin{array}{lllllllll}
A & M & M & A & M & M & A & M A & B
\end{array}\right]
\end{aligned}
$$

and the exponents matrix,

$$
C_{D}=\left[\begin{array}{ccccccccc} 
& A & M & A & M & M & M & M A & M B \\
A & & B & M & M & B & M & B & M B \\
A & A & & M & B & B & B & M & M B \\
M & M & B & & M & M & M & M & B \\
M & M & B & M & & M & M & M & M B \\
M & M & B & A & M & & M & M & M B \\
A & A & M & M A & A & A & & A & B \\
A & A & M & A & A & M & A & & M A \\
B & B & M B & M B & M B & M B & M B & M &
\end{array}\right]
$$

After, applying the proposed algorithm, we obtain the following matrix OD,

Table 2: Resulting O-D matrix using the proposed algorithm.

\begin{tabular}{|c|c|c|c|c|c|c|c|c|}
\hline 0 & 2945.4 & 1142.1 & 1667.2 & 433.1 & 575.7 & 486.1 & 4203.5 & 85.9 \\
\hline 2051.3 & 0 & 381.6 & 557.1 & 289.4 & 192.4 & 324.9 & 351.2 & 28.7 \\
\hline 1467.9 & 1408.6 & 0 & 398.7 & 103.6 & 137.7 & 116.2 & 502.6 & 41.1 \\
\hline 2036.9 & 1954.6 & 757.9 & 0 & 295.3 & 392.6 & 331.5 & 872.9 & 142.7 \\
\hline 1978.7 & 1898.8 & 736.3 & 552.2 & 0 & 381.3 & 322 & 847.9 & 69.3 \\
\hline 1853.7 & 1778.8 & 689.8 & 1034.6 & 268.7 & 0 & 301.7 & 794.4 & 64.9 \\
\hline 4903.1 & 4705 & 1824.4 & 2736.4 & 710.8 & 944.9 & 0 & 2101.1 & 171.8 \\
\hline 8445.1 & 8104 & 3142.4 & 3292.4 & 1710.5 & 1136.9 & 1920 & 0 & 783.6 \\
\hline 1234.6 & 1184.7 & 459.4 & 240.6 & 125 & 166.2 & 140.3 & 350.4 & 0 \\
\hline
\end{tabular}

Which is compared to the original test matrix

Table 3: Original test matrix.

\begin{tabular}{|c|c|c|c|c|c|c|c|c|}
\hline 0 & 2585 & 1155 & 920 & 543 & 460 & 485 & 3247 & 211 \\
\hline 1769 & 0 & 653 & 510 & 322 & 306 & 353 & 538 & 151 \\
\hline 1704 & 1531 & 0 & 519 & 302 & 267 & 295 & 901 & 165 \\
\hline 2282 & 2194 & 1012 & 0 & 465 & 401 & 440 & 844 & 231 \\
\hline 1972 & 1935 & 832 & 687 & 0 & 441 & 496 & 828 & 179 \\
\hline 2049 & 2064 & 946 & 778 & 519 & 0 & 598 & 789 & 212 \\
\hline 3953 & 4127 & 1752 & 1554 & 1009 & 884 & 0 & 1557 & 425 \\
\hline 7308 & 7405 & 3766 & 3018 & 1617 & 1398 & 1556 & 0 & 805 \\
\hline 1447 & 1440 & 1204 & 343 & 263 & 264 & 263 & 329 & 0 \\
\hline
\end{tabular}

We can see that the results for the dominant values of the matrix are approximated; obviously the errors are due to the uncertain information coming from the expert, but still much lower than using the approximation for maximum entropy.

Table 4: Resulting O-D matrix using the maximum entropy method.

\begin{tabular}{|c|c|c|c|c|c|c|c|c|}
\hline 0 & 1181.2 & 1228.4 & 526.2 & 353.4 & 438.1 & 351.4 & 2011.1 & 540.3 \\
\hline 2153.9 & 0 & 1183.9 & 507.2 & 681.1 & 422.2 & 677.4 & 484.6 & 520.8 \\
\hline 1774.2 & 1875.5 & 0 & 417.8 & 280.5 & 347.8 & 279 & 798.3 & 857.9 \\
\hline 1915.8 & 2025.2 & 2106.1 & 0 & 593.9 & 736.3 & 590.7 & 522.6 & 1123.2 \\
\hline 2069.3 & 2187.5 & 2274.9 & 477.7 & 0 & 795.3 & 638 & 564.4 & 606.6 \\
\hline 2001.9 & 2116.2 & 2200.7 & 924.2 & 620.6 & 0 & 617.2 & 546 & 586.8 \\
\hline 1970.7 & 2083.2 & 2166.4 & 909.8 & 610.9 & 757.4 & 0 & 537.5 & 577.7 \\
\hline 3243.1 & 3428.3 & 3565.2 & 1129.6 & 1517.1 & 940.4 & 1508.8 & 0 & 885.8 \\
\hline 3907.4 & 4130.5 & 4295.4 & 680.5 & 913.9 & 1133 & 908.9 & 248.2 & 0 \\
\hline
\end{tabular}


Making a comparison of the percentage of the errors of both methods shows that the method of the proposed algorithm presents a certain amount of errors lower than the highest entropy method. Hereunder is the matrix that arises from the proposed algorithm.

Table 5: Percentage errors using the proposed algorithm.

\begin{tabular}{|c|c|c|c|c|c|c|c|c|}
\hline & $-13,94$ & 1,12 & $-81,22$ & 20,24 & $-25,15$ & $-0,23$ & $-29,46$ & 59,29 \\
\hline$-15,96$ & & 41,56 & $-9,24$ & 10,12 & 37,12 & 7,96 & 34,72 & 80,99 \\
\hline 13,86 & 7,99 & & 23,18 & 65,70 & 48,43 & 60,61 & 44,22 & 75,09 \\
\hline 10,74 & 10,91 & 25,11 & & 36,49 & 2,09 & 24,66 & $-3,42$ & 38,23 \\
\hline$-0,34$ & 1,87 & 11,50 & 19,62 & & 13,54 & 35,08 & $-2,40$ & 61,28 \\
\hline 9,53 & 13,82 & 27,08 & $-32,98$ & 48,23 & & 49,55 & $-0,68$ & 69,39 \\
\hline$-24,03$ & $-14,01$ & $-4,13$ & $-76,09$ & 29,55 & $-6,89$ & & $-34,95$ & 59,58 \\
\hline$-15,56$ & $-9,44$ & 16,56 & $-9,09$ & $-5,78$ & 18,68 & $-23,39$ & & 2,66 \\
\hline 14,68 & 17,73 & 61,84 & 29,85 & 52,47 & 37,05 & 46,65 & $-6,50$ & \\
\hline
\end{tabular}

Meanwhile the matrix that arises from the highest entropy method provides higher percentage errors in some cells, even 100 to 200 percent.

Table 6: Percentage errors using the maximum entropy method.

\begin{tabular}{|c|c|c|c|c|c|c|c|c|}
\hline & 54,31 & $-6,35$ & 42,80 & 34,92 & 4,76 & 27,55 & 38,06 & $-156,07$ \\
\hline$-21,76$ & & $-81,30$ & 0,55 & $-111,52$ & $-37,97$ & $-91,90$ & 9,93 & $-244,90$ \\
\hline$-4,12$ & $-22,50$ & & 19,50 & 7,12 & $-30,26$ & 5,42 & 11,40 & $-419,94$ \\
\hline 16,05 & 7,69 & $-108,11$ & & $-27,72$ & $-83,62$ & $-34,25$ & 38,08 & $-386,23$ \\
\hline$-4,93$ & $-13,05$ & $-173,43$ & 30,47 & & $-80,34$ & $-28,63$ & 31,84 & $-238,88$ \\
\hline 2,30 & $-2,53$ & $-132,63$ & $-18,79$ & $-19,58$ & & $-3,21$ & 30,80 & $-176,79$ \\
\hline 50,15 & 49,52 & $-23,65$ & 41,45 & 39,45 & 14,32 & & 65,48 & $-35,93$ \\
\hline 55,62 & 53,70 & 5,33 & 62,57 & 6,18 & 32,73 & 3,03 & & $-10,04$ \\
\hline$-170,03$ & $-186,84$ & $-256,76$ & $-98,40$ & $-247,49$ & $-329,17$ & $-245,59$ & 24,56 & \\
\hline
\end{tabular}

\section{CONCLUSIONS}

In this paper the potential and effectiveness of this new methodology has been proven. Clearly, the obtained matrix has an error of $10 \%$ compared to the original matrix. However, these results are reasonable considering the level of uncertainty coming from the information provided by the expert.

As a guideline for future research, it is interesting to improve the fuzzy inference engine, since the used in this test was quite simple. Here, other sources of information can be incorporated without limiting the information just to one expert.

\section{ACKNOWLEDGEMENTS}

The authors of this paper want to express their gratitude to the National Secretary of Science and Technology (SENACYT) of the government of the Republic of Panama for funding this study through the R \& D project "Methodologies and Performance Indicators of Railway Transport Systems" adjudged by a call for the promotion of R\&D activities (MDEPRB09-001). Additionally, they want to thank the Research Centre on Railway Technologies (CITEF) of Universidad Politécnica de Madrid (Spain).

\section{REFERENCES}

Cheng, Y.H. y Yang, Li-An, 2009. A Fuzzy Petri Nets approach for railway traffic control in case of abnormality: Evidence from Taiwan railway system. Expert Systems with Applications 36 (2009) 80408048.

Lindaren, R. y Tantiyanugulchai, S., 2003. Microscopic Simulation of Traffic at a Suburban Interchange.

Watson, J. R. y Prevedouros, P. D., 2006. Derivation of Origin-Destination Distributions from Traffic Counts Implications for Freeway Simulation. Transportation Research Record: Journal of the Transportation Research Board,No. 1964, Transportation Research Board of the National Academies, Washington, D.C., 2006, pp. 260-269.

S. Kikuchi y D. Miljkovic., 1999. Method To Preprocess Observed Traffic Data for Consistency Application of Fuzzy Optimization Concept. Transportation Research Record 1679 Paper No. 99-0129 73.

Aldian, A y Taylor, M., 2003. Fuzzy multicriteria analysis for inter-city travel demand modelling. Journal of the Eastern Asia Society for Transportation Studies, Vol. 5, October, 2003.

C. H. Murat., 2010. Sample size needed for calibrating trip distribution and behaviour of the gravity model. Journal of Transport Geography 18,183-190.

C. Xie, K.M. Kockelman y S.T. Waller., 2010. A maximum entropy method for subnetwork origindestination 1 trip matrix estimation. The 89th Annual Meeting of the Transportation Research Board, January 2010 in Washington, DC, and for publication in Transportation Research Record. 2010. 